\title{
TRANSPORTE, PATOGENICIDADE E TRANSMISSIBILIDADE DE FUNGOS ASSOCIADOS ÀS SEMENTES DE PINHÃO MANSO ${ }^{1}$
}

\author{
DANILA ALVES CORRÊA DE SÁ2, GIL RODRIGUES DOS SANTOS ${ }^{3}$, GLEIBER QUINTÃO FURTADO ${ }^{4}$, \\ EDUARDO ANDRÉA LEMUS ERASMO ${ }^{5}$, ILDON RODRIGUES DO NASCIMENTO ${ }^{6}$
}

\begin{abstract}
RESUMO- Objetivou-se determinar a taxa de transporte de população de fungos associados às sementes de pinhão manso, a patogenicidade desses microrganismos a plântulas e frutos e a transmissibilidade fruto-semente e semente-plântula. Avaliaram-se a taxa de transporte, por meio de blotter test, de sementes produzidas nos estados de Minas Gerais, São Paulo, Bahia e Tocantins. As sementes foram submetidas aos tratamentos: sem desinfestação com tegumento (SDCT), sem desinfestação sem tegumento (SDST), com desinfestação com tegumento (CDCT) e com desinfestação sem tegumento (CDST). A incidência (\%) dos fungos foi avaliada sob microscópio estereoscópico binocular. Para o teste de patogenicidade em plântulas e frutos inocularam-se suspensões de $10^{6}$ esporos e discos de BDA com micélio, respectivamente. Para os fungos fitopatogênicos avaliaram-se a transmissibilidade fruto-semente e semente-plântula. O tratamento SDCT permitiu a deteç̧ão de maior número de fungos. Os fungos identificados foram Colletotrichum gloeosporioides, C. capsici, Curvularia sp., Verticillium sp., Fusarium sp., Penicillium sp., Aspergillus sp., A. niger e Rhizopus sp. Apenas as espécies de Colletotrichum são patogênicas às plântulas e frutos. Para ambas espécies há transmissibilidade fruto-semente, entretanto não é observada transmissão semente-plântula.
\end{abstract}

Termos para indexação: Jatropha curcas L., patologia de sementes, Colletotrichum

\section{TRANSPORT, PATHOGENICITY AND TRANSMISSIBILITY OF FUNGI ASSOCIATED WITH PHYSIC NUT SEEDS}

\begin{abstract}
The objective of the present study was to determine the transport rate of a fungus population associated with Jatropha curcas seeds and the pathogenicity of these microrganisms to seedlings and fruit and also the fruit-seed and seed-seedling transmissibility. The transport rate of seeds produced in Minas Gerais, São Paulo, Bahia and Tocantins states was evaluated using the blotter test. The seeds were submitted to the following treatments: without disinfestation, with tegument (SDCT), without disinfestation, without tegument (SDST), with disinfestation, with tegument (CDCT) and with disinfestations, without tegument (CDST). The incidence (\%)
\end{abstract}

${ }^{1}$ Submetido em 11/09/10. Aceito para publicação em 03/05/2011. Parte da dissertação de Mestrado do primeiro autor apresentada a Universidade Federal do Tocantins (UFT).

${ }^{2}$ Eng. Agr., Mestre do Programa em Agroenergia da UFT, campus universitário de Palmas, 77001-090, Palmas, TO, Brasil, danilasa@ hotmail.com

${ }^{3}$ Eng. Agr., Dr., Professor Adjunto, Departamento de Fitopatologia, Bolsista de Produtividade em Pesquisa - CNPQ, UFT, Caixa Postal 66,
CEP 77402-970, Gurupi, TO, Brasil. gilrsan@uft.edu.br

${ }^{4}$ Eng. Flo., Dr., Professor Adjunto, Departamento de Fitopatologia, UFV, CEP 36570-000, Viçosa, gfurtado@ufv.br

${ }^{5}$ Eng. Agr., Dr., Professor Adjunto, UFT, Caixa Postal 66, CEP $77402-$ 970, Gurupi, erasmolemus@uol.com.br

${ }^{6}$ Eng. Agr., Dr., Professor Adjunto, UFT, Caixa Postal 66, CEP 77402970, Gurupi, ildon@uft.edu.br 
of the fungi was assessed using a binocular stereomicroscope. Suspensions of $10^{6}$ spores and PDA discs with mycelium were inoculated for the pathogenicity tests on seedlings and fruits, respectively. For the phytopathogenic fungi, the fruit-seed and seed-seedling transmissibility were evaluated. The SDCT treatment allowed the detection of a greater number of fungi. The identified fungi were Colletotrichum gloeosporioides, C. capsici, Curvularia sp., Verticillium sp., Fusarium sp., Penicillium sp., Aspergillus sp., A. Niger, Rhizopus sp. Only the Colletotrichum species is pathogenic to seedlings and fruit. For both species there is fruit-seed transmissibility but no seedseedling transmission.

Index terms: Jatropha curcas L., seed pathology, Colletotrichum

\section{INTRODUÇÃO}

Atualmente a busca por fontes renováveis de energia tem se intensificado principalmente devido à escassez $\mathrm{e}$ elevação do preço do petróleo e as preocupações com as mudanças climáticas globais. Nesse cenário a espécie Jatropha curcas L., popularmente conhecida por pinhão manso, é considerada promissora para a produção de biodiesel, produzindo em torno de $38 \%$ de óleo em suas sementes (Dias et al., 2007).

Apesar de ser considerada uma planta rústica, adaptada a condições edafoclimáticas marginais, o pinhão manso necessita da aplicação de tecnologias de cultivo (adubação, controle de pragas e doenças, práticas de manejo e outros) para apresentar níveis econômicos de produção de frutos (Laviola e Dias, 2008).

As sementes dessa Euforbiácea são constituídas por um tegumento denso e escuro, protegendo o embrião. Os frutos são do tipo cápsula ovóide, com 1,5 a 3,0 cm de diâmetro, trilocular, sendo uma semente por lóculo (Saturnino et al., 2005; Dias et al., 2007).

De uma maneira geral, patógenos associados às sementes são transportados de duas maneiras: infecção ou infestação (contaminação). A infecção implica que o patógeno é transportado internamente, incrustrado nos tecidos da semente. Quando um patógeno é transportado passivamente, ele é um contaminante ou infestante. Neste caso, o patógeno localiza-se sobre a superfície da semente (Agarwal e Sinclair, 1987).

A partir de janeiro de 2008, o Ministério da Agricultura, Pecuária e Abastecimento (MAPA), através da Instrução Normativa n.4, autorizou a inscrição da espécie $J$. curcas no Registro Nacional de Cultivares (RNC). A proibição de cultivo da espécie decorria, sobretudo, da ausência de pesquisas sobre o desempenho agronômico da cultura e, entre elas, a ausência de estudos básicos de suas sementes (Pinto et al., 2009). Existem poucos estudos relacionados à qualidade fitossanitária de sementes de pinhão manso no Brasil e mundo. Com base nisso, o presente trabalho teve como objetivo realizar um levantamento da população fúngica transportada interna e externamente às sementes, a determinação da patogenicidade desses microrganismos a frutos e plântulas e a sua transmissibilidade fruto-semente e semente-plântulas.

\section{MATERIAL E MÉTODOS}

Os experimentos foram conduzidos no Laboratório de Fitopatologia e em casa de vegetação da Universidade Federal do Tocantins, campus de Gurupi-TO.

\section{Transporte de fungos associados às sementes}

O levantamento da população fúngica associada às sementes de pinhão manso foi realizado por meio do método do papel de filtro ou "blotter test" (Brasil, 2009). Para isso, utilizaram-se caixa acrílica tipo Gerbox, desinfestada com álcool $70 \%$, contendo em seu interior duas folhas de papel de filtro esterilizadas e umedecidas com $50 \mathrm{~mL}$ de água destilada estéril com adição de $100 \mathrm{ppm}$ de sulfato de estreptomicina. As sementes de pinhão manso utilizadas foram provenientes dos estados da Bahia, Minas Gerais, São Paulo e Tocantins, sendo todas produzidas no ano agrícola de 2008.

As sementes provenientes de cada lote foram submetidas aos seguintes tratamentos: sem desinfestação com tegumento (SDCT), sem desinfestação sem tegumento (SDST), com desinfestação com tegumento (CDCT) e com desinfestação sem tegumento (CDST). As desinfestações foram realizadas emergindo as sementes em uma sequência de solução de álcool $70 \%$ 
por 30 segundos, hipoclorito de sódio $2 \%$ por 2 minutos e posteriormente água destilada esterilizada. As sementes não desinfestadas foram lavadas em água destilada esterilizada durante 1 minuto. A remoção do tegumento foi realizada com auxílio de um bisturi esterilizado.

As sementes foram dispostas individualmente e, posteriormente, acondicionadas em câmara de crescimento, tipo BOD, sob temperatura de $25{ }^{\circ} \mathrm{C}$ e fotoperíodo de 12 horas durante sete dias. A avaliação foi realizada sob microscópio estereoscópico, aos cinco dias após a incubação. Para identificação dos fungos lâminas foram preparadas e visualizadas em microscópio de luz, anotando-se a incidência (\%) de todos os fungos encontrados. Os fungos foram isolados e cultivados em meio de cultura batata-dextrose-ágar (BDA). As espécies de Colletotrichum gloeosporioides e C. capsici foram identificadas no Laboratório de Micologia da ESALQ/USP, por meio de análise morfológica, cultura e molecular.

Os experimentos seguiram o delineamento inteiramente casualizado, com dez repetições, cada repetição consistiu de um Gerbox contendo dez sementes.

\section{Teste de patogenicidade em plântulas e frutos}

A patogenicidade dos fungos transportados pelas sementes foi avaliada por meio de inoculações na parte aérea de plântulas e frutos dessa espécie oleaginosa.

Para obtenção das plântulas, 20 sementes foram desinfestadas, conforme descrito no item anterior, e semeadas em bandejas plásticas contendo substrato Plantimax ${ }^{\circledR}$ esterilizado. Após 20 dias da realização da semeadura, a parte área de plântulas sadias foi pulverizada com suspensão de $10^{6}$ esporos $/ \mathrm{mL}$. As suspensões foram preparadas a partir de colônias de fungos cultivadas em meio BDA e incubadas por oito dias em BOD, sob temperatura de $25{ }^{\circ} \mathrm{C}$ e fotoperíodo de 12 horas. As plântulas inoculadas foram acondicionadas em câmara úmida e escura por $72 \mathrm{~h}$. Após esse período, as mesmas foram transferidas para casa de vegetação, onde permaneceram por sete dias.

As avaliações dos sintomas foram realizadas dez dias após a inoculação. Posteriormente, realizou-se o isolamento indireto do fungo, de acordo com Alfenas e Mafia (2007), com a finalidade de confirmação do agente causal. $\mathrm{O}$ experimento seguiu o delineamento inteiramente casualizado (DIC), com dez tratamentos (fungos) e 20 repetições. Cada repetição consistiu em uma plântula.

Para o teste de patogenicidade em frutos utilizaramse apenas os fungos que foram capazes de causar doença em plântulas, sendo Colletotrichum gloeosporioides e $C$. capsici. Utilizaram-se frutos em estádios semi-maduros, com coloração verde-amarela, colhidos no município de Gurupi-TO. Os frutos foram submetidos a uma assepsia com hipoclorito de sódio (1\%) e, posteriormente, lavados em água estéril. Esses foram perfurados superficialmente com o auxílio de uma agulha esterilizada. Posteriormente, foi depositado um disco de BDA de cinco $\mathrm{mm}$ de diâmetro, contendo crescimento micelial de cada fungo. Utilizaram-se como inóculo estruturas de fungos com dez dias. Os frutos inoculados foram acondicionados em Gerbox, conforme metodologia descrita para a incubação das sementes. Os experimentos foram instalados em delineamento inteiramente casualizado, com dez repetições, sendo cada repetição composta por dois Gerbox, contendo cada um cinco frutos. Os frutos foram avaliados diariamente, por um período de 10 dias, quanto à incidência de sintomas. Para a confirmação do agente causal, foi realizado o isolamento do fungo de acordo com a metodologia descrita para plântulas.

\section{Transmissibilidade fruto-semente e semente- plântula}

Para os fungos que foram patogênicos a frutos, investigaram-se a transmissão as suas sementes. Para isso, os frutos foram inoculados e incubados conforme descrito anteriormente para o teste de patogenicidade em frutos. Aos 10 dias após a inoculação, as sementes foram retiradas do interior dos frutos e submetidas ao método do papel de filtro ou "blotter test" e incubadas durante três dias. As sementes foram analisadas individualmente sob microscópio estereoscópico e para identificação do fungo lâminas foram preparadas e visualizadas ao microscópio de luz. Os fungos foram isolados e cultivados em meio de cultura BDA para confirmação. Posteriormente, avaliaram-se a incidência (\%) de sementes contaminadas com o fungo inoculado em frutos. O delineamento experimental foi inteiramente casualizado. Para cada fungo testado foi utilizado três repetições, sendo cada repetição composta por cincos frutos. Os frutos testemunhas foram perfurados, porém receberam discos de BDA sem nenhuma estrutura fúngica.

Quando houve comprovação da transmissão fruto-semente, outros frutos foram inoculados, e as sementes obtidas foram semeadas para observação da transmissibilidade semente-plântula. As sementes infestadas foram semeadas em bandejas plásticas com 28 x $43 \mathrm{~cm}$ de dimensão, contendo substrato Plantimax ${ }^{\circledR}$ 
esterilizado. As bandejas foram acondicionadas em casa de vegetação. A umidade do substrato foi mantida por meio de irrigações diárias. Aos dez dias após a semeadura avaliaram-se a incidência (\%) de plântulas sintomáticas, observando cotilédones, hastes ou folhas. Para confirmação do agente causal da doença foi realizado o isolamento indireto do fungo.

Foram utilizadas, para cada fungo, quatro repetições, sendo uma bandeja com dez sementes uma repetição. Para cada fungo testado, utilizaram-se como testemunha uma bandeja contendo dez sementes provenientes de frutos não inoculados.

\section{RESULTADOS E DISCUSSÃO}

Transporte de fungos associados às sementes de pinhão manso.

De acordo com a Tabela 1, a detecção de populações de fungos em sementes de pinhão manso só foi possível naquelas que foram submetidas aos tratamentos que preconizaram a manutenção do tegumento, onde naquelas desinfestadas a incidência de fungos foi maior. Essa observação indica que o tegumento funciona como uma barreira física à infecção desses microrganismos em sementes dessa oleaginosa.

TABELA 1. Taxa de transporte (\%) de fungos associados a sementes de pinhão manso (Jatropha curcas) submetidas aos tratamentos sem desinfestação com tegumento (SDCT), sem desinfestação sem tegumento (SDST), com desinfestação com tegumento (CDCT) e com desinfestação sem tegumento (CDST), originárias dos estados de Minas Gerais, São Paulo, Bahia e Tocantins.

\begin{tabular}{|c|c|c|c|c|c|}
\hline \multirow{2}{*}{ Origem } & \multirow{2}{*}{ Fungos } & \multicolumn{4}{|c|}{ Taxa de transporte $(\%)$} \\
\hline & & SDCT & SDST & $\mathrm{CDCT}$ & CDST \\
\hline \multirow{7}{*}{ Minas Gerais } & Colletotrichum capsici & 7 & - & - & - \\
\hline & Curvularia sp. & 3 & - & - & - \\
\hline & Verticillium sp. & 6 & - & - & - \\
\hline & Fusarium sp. & 25 & - & - & - \\
\hline & Penicillium sp. & 7 & - & - & - \\
\hline & Aspergillus sp. & 6 & - & 2 & - \\
\hline & Alternaria sp. & 6 & - & - & - \\
\hline \multirow{6}{*}{ São Paulo } & Curvularia sp. & 3 & - & - & - \\
\hline & Verticillium sp. & 87 & - & - & - \\
\hline & Fusarium sp. & 7 & - & 1 & - \\
\hline & Aspergillus sp. & 11 & - & 2 & - \\
\hline & Alternaria sp. & 11 & - & - & - \\
\hline & Rhizopus sp. & 2 & - & 2 & \\
\hline \multirow{4}{*}{ Tocantins } & Aspergillus sp. & - & - & 2 & - \\
\hline & A. Níger & - & - & 2 & - \\
\hline & Verticillium sp. & - & - & - & - \\
\hline & Penicillium sp. & - & - & 1 & - \\
\hline \multirow{9}{*}{ Bahia } & C. gloeosporioides & 10 & - & - & - \\
\hline & Curvularia sp. & 3 & - & - & - \\
\hline & Verticillium sp. & 4 & - & - & - \\
\hline & Fusarium sp. & 7 & - & - & - \\
\hline & Penicillium sp. & 10 & - & - & - \\
\hline & Aspergillus sp. & 50 & - & 2 & - \\
\hline & $A$. niger & 2 & - & - & - \\
\hline & Rhizopus sp. & 3 & - & - & - \\
\hline & Alternaria sp. & 60 & - & - & - \\
\hline
\end{tabular}

- Ausência de fungos 
Para o gênero Colletotrichum determinadas espécies ficam externamente, outras internamente na semente. Estudos histopatológicos em sementes de soja infectadas por C. truncatum mostram que este fungo usualmente coloniza o tegumento da semente, formando acérvulos, mas raramente os tecidos do embrião (Schneider et al. 1974; Rodriguez-Marcano e Sinclair, 1978). No entanto, a espécies $C$. kikuchii causa mancha púrpura em sementes de soja, porém penetra o tegumento através do funículo (Sinclair e Backman, 1989). Em sementes de algodão inoculada com Colletotrichum gossypii var. cephalosporioides, Tanaka et al. (1989) observaram que o contato das sementes com o inóculo, a partir de 12 até 48 horas, resultou em associação tipo infecção, decorrente da penetração do fungo pelo tegumento.

Os fungos transportados pelas sementes proveniente de Minas Gerais foram Colletotrichum capsici, Curvularia, Verticillium, Fusarium, Penicillium, Aspergillus e Alternaria. Com exceção de Colletotrichum e inclusão de Rhizopus, todos os demais fungos foram encontrados associados também às sementes oriundas de estado de São Paulo. Para as sementes provenientes do estado do Tocantins, apenas os fungos dos gêneros Aspergillus, Verticillium, Penicillium e A. niger foram detectados.

Santos et al. (2009) ao avaliar a qualidade fitossanitária de sementes de pinhão manso produzidas no Norte de Minas Gerais, detectaram os gêneros Fusarium, Aspergillus, Penicillium e Colletotrichum.

As maiores incidências de fungos encontrados nas sementes provenientes do estado de Minas Gerais (Tabela 1) foram os gêneros Fusarium (25\%) e Colletotrichum (7\%). Já para as sementes oriundas de São Paulo os fungos mais frequentes foram Verticillium (87\%), Alternaria e Aspergillus, ambos com incidência de $11 \%$. Para aquelas produzidas no estado do Tocantins destacaram os fungos Aspergillus (52\%) e Penicillium (49\%). Os fungos com maiores incidências em sementes do estado da Bahia foram Aspergillus (50\%), Alternaria (60\%), Colletotrichum gloeosporioides e Penicillium, ambos com $10 \%$.

Os gêneros Fusarium e Aspergillus também foram encontrados com alta incidência em sementes de pinhão manso em trabalho realizado por Melo et al. (2007) e Neves et al. (2009), o que corrobora os resultados aqui encontrados.

Kobayasti et al. (2009) avaliaram a incidência de fungos fitopatogênicos em sementes de pinhão manso, produzidas no Mato Grosso, colhidas em frutos secos e maduros, com e sem desinfestação. Em todos os tratamentos foi possível a detecção de diversos fungos, fitopatogênicos e não-fitopatogênicos. A espécie Colletotrichum gloeosporioides foi encontrada em sementes não desinfestadas produzidas em frutos secos.

Os fungos Aspergillus spp., Rhizopus e Penicillium spp. estão relacionados na maioria das vezes às condições inadequadas de armazenagem, ocorrendo geralmente após a colheita ou durante o armazenamento das sementes (Dhingra et al., 1980; Machado, 1988).

\section{Teste de patogenicidade em plântulas e frutos}

Entre os fungos transportados externamente às sementes de pinhão manso apenas as espécies Colletotrichum gloeosporioides e C. capsici foram patogênicas às plântulas e frutos (Tabela 2 e Figura 1). Os demais gêneros, alguns conhecidos como fitopatogênicos, não foram aptos a incitarem doenças nas condições experimentais utilizadas.

TABELA 2. Patogenicidade a plântulas e frutos de pinhão manso de fungos transportados por sementes de pinhão manso.

\begin{tabular}{lcc}
\hline \multicolumn{1}{c}{ Fungos } & Plântula & Fruto \\
\hline Colletotrichum capsici & + & + \\
Colletotrichum gloeosporioides & + & + \\
Curvularia & - & - \\
Verticillium & - & - \\
Fusarium & - & - \\
Penicillium & - & - \\
Aspergillus & - & - \\
Alternaria & - & - \\
A. níger & - & - \\
Rhizopus & - & - \\
\hline
\end{tabular}

(+) Fungo patogênico, (-) Fungo não patogênico

Os sintomas incitados em plântulas de pinhão manso pelas espécies Colletotrichum capsici e C. gloeosporioides foram idênticos, consistiram de lesões irregulares com coloração castanho-clara a castanho-escura acompanhada de necrose das nervuras, culminando com a necrose dos tecidos lesionados (Figura1A e B). Estes sintomas são semelhantes aos citados por (Franco e Gabriel 2008; Freire e Parente 2006). 

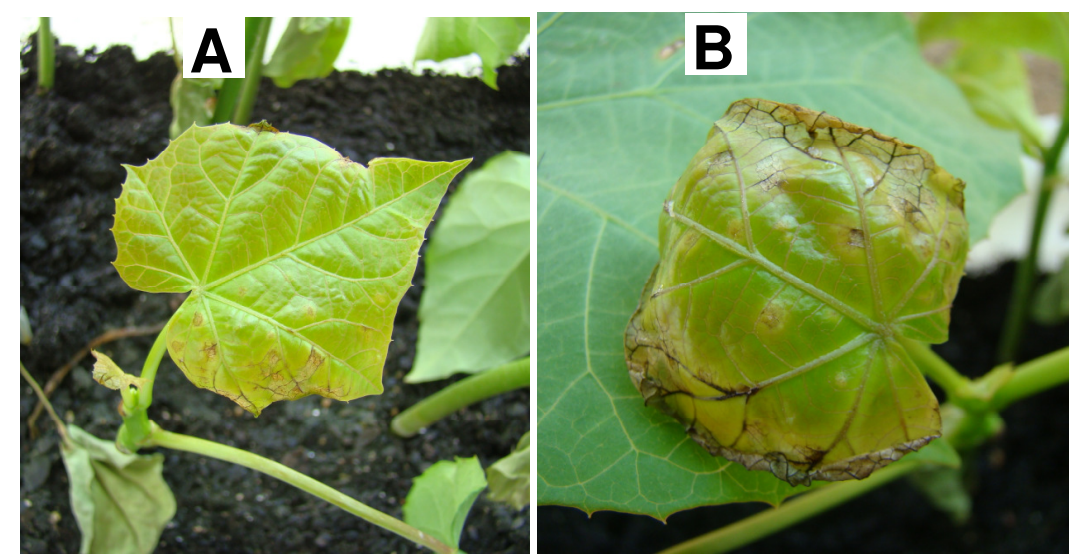

FIGURA 1. Sintomas de antracnose ocasionados por Colletotrichum gloeosporioides e C. capsici em plântulas de pinhão manso. Aspecto da lesão dois dias (A) e cinco dias após a inoculação (B).

Transmissibilidade fruto-semente e sementeplântula

A inoculação de frutos maduros de pinhão manso com os fungos Colletotrichum capsici e C. gloeosporioides proporcionou $100 \%$ de transmissão destes patógenos as suas sementes (Tabela 3, Figura 2). No entanto, não houve transmissibilidade semente-plântula para estes fungos. Provavelmente, esta falha na transmissão seja decorrente de dois fatores, à rápida liberação do tegumento pelas sementes durante o processo de germinação e ao fato destes fungos terem sido detectados apenas externamente as sementes de pinhão manso (Tabela 1).

TABELA 3. Transmissibilidade (\%) fruto-semente e semente-plântula de espécies de Colletotrichum em pinhão manso.

\begin{tabular}{ccc}
\hline \multirow{2}{*}{ Fungos } & \multicolumn{2}{c}{ Transmissão (\%) } \\
\cline { 2 - 3 } & Fruto-semente & Semente-plântula \\
\hline Colletotrichum capsici & 100 & - \\
Colletotrichum gloeosporioides & 100 & - \\
\hline
\end{tabular}

(-) sem transmissão

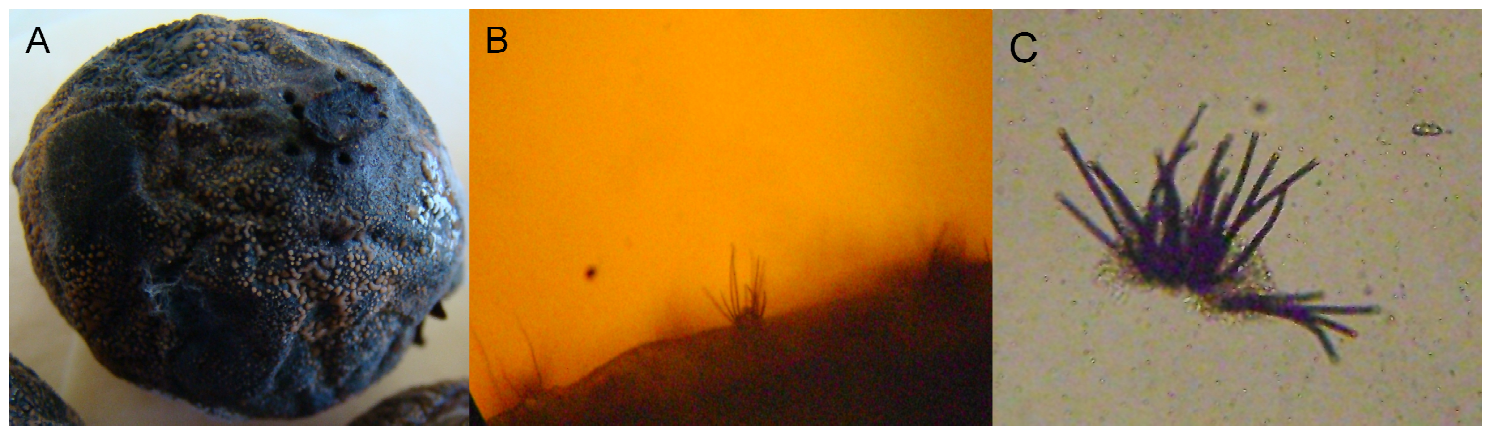

FIGURA 2. Antracnose em frutos e sementes de pinhão manso decorrente da inoculação de frutos com as espécies Colletotrichum capsicie C.gloeosporioides. (A)Frutonecrosadocom esporulação alaranjada nasuperfície, (B) Acérvulos na superfície de semente infectada, (C) estrutura acervular de Colletotrichum. 
Entre os agentes patogênicos, os fungos são os mais ativos, tendo uma maior habilidade em penetrar diretamente nos tecidos vegetais e se alojarem internamente. O inóculo fúngico pode ser transportado via semente, na forma de micélio e/ou de esporos, mas a taxa de transmissão do patógeno, depende fundamentalmente da quantidade e localização do inóculo na semente. A presença do patógeno no embrião da semente é a maneira mais eficiente de se garantir a infecção da plântula que dela será originada (Neergaard, 1979; Machado, 1988; Menten, 1991; Tanaka e Machado, 1985).

Dessa forma, o tegumento da semente de pinhão manso parece funcionar como uma barreira natural impedindo a penetração (infecção) de patógenos a sementes. Novos estudos serão necessários para elucidar essa proteção.

\section{CONCLUSÕES}

Os fungos transportados pelas sementes do pinhão manso encontram-se externamente ao tegumento.

As espécies C. capsici e C. gloeosporioides são patogênicos a plântulas e frutos de pinhão manso

Os fungos C. capsici e C. gloeosporioides são transmitidos de frutos a sementes, mas não são de sementes para plântulas de pinhão manso.

\section{REFERÊNCIAS}

AGARWAL, V.K.; SINCLAIR, J.B. Principles of seed pathology. Boca Raton: CRC Press, 1987. v.1, 175p.

ALFENAS, A.C.; FERREIRA, F.A.; MAFIA, R.G.; GONÇALVES, R.C. Isolamentos de fungos fitopatogênicos. In: ALFENAS, A.C.; MAFIA, R.G. Métodos em Fitopatologia. Viçosa: Editora UFV, 2007, p.53-90, 2007.

BRASIL. Ministério da Agricultura, Pecuária e Abastecimento. Regras para análise de sementes. Ministério da Agricultura, Pecuária e Abastecimento. Secretaria de Defesa Agropecuária. Brasília, DF: MAPA/ ACS, 2009. 395p.

DHINGRA, O.D.; MUCHOVEJ, J.J.; CRUZ FILHO, J. Tratamento de sementes (controle de patógenos). Viçosa: UFV, Imprensa Universitária, 1980. 121p.

DIAS, L.A.S.; LEME, L.P.; LAVIOLA, B.G.; PALLINI, A.; PEREIRA, O.L.; DIAS, D.C.F.S.; CARVALHO, M.; MANFIO, C.E.; SANTOS, A.S.; SOUZA, L.C.A.; OLIVEIRA, T.S.; PRETTI, L.A. Cultivo de pinhão manso (Jatropha curcas L.): para produção de óleo combustível.
Viçosa: Editora UFV, 2007. 40p.

FRANCO, D.A.S.; GABRIEL, D. Aspectos fitossanitários na cultura do pinhão manso (Jatropha curcas L.) para produção de biodiesel. In: REUNIÃO ANUAL DO INSTITUTO BIOLÓGICO, 21., 2008, São Paulo. Anais... São Paulo: IB, 2008, p.63-64.

FREIRE, F.C.O.; PARENTE, G.B. As doenças da jatrofa (Jatropha curcas L. e J. podagrika Hooke.) no estado do Ceará. Fortaleza: Embrapa Agroindústria Tropical, 2006. 3p. (Embrapa Agroindústria Tropical. Comunicado técnico, 120).

KOBAYASTI, L.; ADORIAM, A.I.; NETO, V.B.P.; ALVES C.Z.; ZUFFO, M.C.R. Levantamento da incidência de fungos potencialmente patogênicos em sementes de pinhão manso. In: CONGRESSO BRASILEIRO DE PESQUISA EM Pinhão manso, I, 2009, Brasília. Anais... Brasília, DF: Embrapa Agroenergia, 2009, n.71. CDROM.

LAVIOLA, B.G.; DIAS, L.A.S. Teor e acúmulo de nutrientes em folhas e frutos de pinhão manso. Revista Brasileira de Ciência Solo, v.32, n.5, p.1969-1975, 2008.

MACHADO, J.C. Patologia de sementes: fundamentos e aplicações. Brasília:, MEC/ESAL-/FAEPE, 1988. 107p.

MELO, M.F.V.; SANTOS, H.O.; SILVA-MANN, R.; MESQUITA, J.B. 2007. Fungos associados a sementes de pinhão manso (Jatropha curcas L.). $\leq$ http://www. biodiesel.gov.br/docs/congresso2007/agricultura $>$. Acesso em: 01 abr. 2009.

MENTEN, J.O.M. Patógeno em sementes: detecção, dano e controle químico. Piracicaba: FEALQ/ESALQ, 1991. $312 p$.

NEERGAARD, P. Seed pathology. London: Mac Millan, 1979. 839p.

NEVES, W.S.; PARREIRA, D.F.; FERREIRA, P.A.; LOPES, E.A. Avaliação fitossanitária de sementes de pinhão manso provenientes dos vales do Jequitinhonha e Mucuri. Revista Trópica - Ciências Agrárias e Biológicas. v.3, n.2, p.17, 2009.

PINTO, T.L.F.; MARCOS FILHO, J.; FORTI, V.A. CARVALHO, C.; GOMES JÚNIOR, F.G.Avaliação da viabilidade de sementes de pinhão manso pelos testes de tetrazólio e de raios X. Revista Brasileira de Sementes, v.31, n.2, p.195-201, 2009.

RODRIGUEZ - MARCANO, A.; SINCLAIR, J.B. Fruiting structures of Colletotrichum dematium var. truncata and Phomopsis sojae formed in soybean seeds. Plant Disease 
Reporter, v.62, n.10, p.873-876, 1978.

SANTOS, A.A.; CALDEIRA, N.C.A.; AQUINO, C.F.; SALES, N.P.L.; MARANGON, B.; BRANDÃO JÚNIOR, D.S. Avaliação da qualidade sanitária das sementes de pinhão manso oriundas do norte de Minas Gerais. In: CONGRESSO BRASILEIRO DE FITOPATOLOGIA, 42., 2009, Rio de Janeiro. Anais... Brasília, DF: SBF, 2009.

SATURNINO, H.M.; PACHECO, D.D.; KAKIDA, J.; TOMINAGA, N.; GONÇALVES, N.P. Cultura do pinhão manso (Jatropha curcas L.). Informe Agropecuário, v.26, n.229, p.44-78, 2005.

SINCLAIR, J.B.; BACKMAN, P.A. Compendium of soybean diseases. 3.ed. Saint Paul: The American Phytopathology Society, 1989. 106p.
SCHNEIDER, R.W.; DHINGRA, D.D.; NICHOLSON, J.F.; SINCLAIR, J.B. Colletotrichum truncatum borne within the seed coat of soybean. Phytopathology, v.64, n.1, p.154-155, 1974.

TANAKA, M.A.S.; MACHADO, J.C. Patologia de sementes. Informe Agropecuário, v.11, n.122, p.40-46, 1985.

TANAKA, M.A.S.; MENTEN. J.O.M.; MARIANNO, M.I.A. Inoculação artificial de sementes de algodão com Colletotrichum gossypii var. cephalosporioides e infecção das sementes em função do tempo de exposição ao patógeno. Summa Phytopathologica, v.15, n.2, p.232-237, 1989. 